\title{
Optimized electrochemical biosensor for human prostatic acid phosphatase
}

\author{
Flávio C. Bedatty Fernandes, Paulo R. Bueno* \\ Chemistry Institute, São Paulo State University, Nanobionics Group, CP14800-900 Araraquara, São Paulo, Brazil ${ }^{1}$
}

\section{A R T I C L E I N F O}

\section{Article history:}

Received 20 January 2017

Received in revised form 11 May 2017

Accepted 6 June 2017

Available online 19 June 2017

\section{Keywords:}

Immittance spectroscopy

Electrochemical impedance spectroscopy

Prostate cancer

Human prostatic acid phosphatase

Label-free sensing

Electrochemical biosensor

\begin{abstract}
A B S T R A C T
An innovative and sensitive label-free electrochemical biosensor platform for detecting human prostatic acid phosphatase ( $h \mathrm{PAP}$ ) is proposed herein. The methodology uses impedance-derived immittance functions (ImFs) for monitoring the interfacial changes in a $h \mathrm{PAP}$ bio-recognition layer constructed using a self-assembled monolayer of PEGlate thiol (low-fouling materials) and 11-ferrocenyl undecanethiol (redox probe confined). We evaluated the response toward the target primarily in phosphatase-buffered saline, at varying concentrations and across a wide range of frequencies. The optimum frequency for each $\operatorname{ImF}$ parameter was selected based on its sensitivity and linearity within a target concentration ranging from $20 \mathrm{pM}$ to $5000 \mathrm{pM}$ and a limit of detection (LoD) below the cut-off value for this cancer biomarker $(10 \mathrm{pM})$. The best $\operatorname{ImF}$ selected (inverse of imaginary capacitance, $\left.1 / \mathrm{C}^{\prime \prime}\right)$ was then successfully used to monitor $h$ PAP in human blood serum and showed a linear range from $50 \mathrm{pM}$ to $10^{4} \mathrm{pM}$, with an LoD of $11.2 \pm 2.6 \mathrm{pM}$. Additionally, both the assay and analysis of the results can be performed in a few minutes without the need to add an interfering redox probe to the biological samples, thus providing a rapid, sensitive, and label-free test for prostate cancer.
\end{abstract}

(c) 2017 Elsevier B.V. All rights reserved.

\section{Introduction}

Electrodes modified with self-assembled monolayers (SAMs) containing redox-active molecules and receptive species have been used as electrochemical platforms in order to develop analytical methods for detecting clinically relevant bio-molecules (biomarkers) [1-7]. In previous works, using this redox configuration, the detection of the target was related to variation in the redox capacitance $\left(C_{r}\right)$ signal. This depends on the charging/discharging process originating from electron transfer associated with the redox centres contained in the molecular bio-recognition layer. The great advantage of this configuration is that no signal amplifiers in the electrolyte or labels in the target are required [1-7]; thus, it offers a simple, sensitive, fast, and low-cost sensorial method that is especially suitable for point-of-care diagnosis [1-7]. The $C_{r}$ signal is measured using an impedance-derived capacitance technique, known as electrochemical capacitance spectroscopy (ECS) [8-11], and then used to monitor the specific interactions when the electrode surface is functionalized with an appropriate receptor for a given target. Recently, a more sophisticated method of data

\footnotetext{
* Corresponding author.

E-mail address: prbueno@iq.unesp.br (P.R. Bueno).

1 www.nanobionics.pro.br.
}

processing based on immittance function concepts (ImFs) [12,13] has been applied to redox-capacitive sensing, which enormously reduces the time taken to perform assays and, in addition, improves the sensitivity and limit of detection (LoD) compared with all other impedimetric data-processing methods reported in the literature $[12,13]$. The immittance-function approach enables the user to select the most responsive frequency range for interfacial changes, individually evaluating the performance of each $\operatorname{Im} F$ parameter, and then use it to run quick measurements within limited frequency ranges or even at single frequencies (Scheme 1 shows an overview of this procedure) [12,13]. Therefore, electrochemical biosensors using ImFs, if combined with standard microfabrication, microfluidic formats, high-affinity/specificity receptors, and effective surface engineering, are able to provide low-cost, high-throughput, and high-innate-sensitivity electroanalysis configurations with the capability to simultaneously quantify multiple biomarker targets in real samples using a single device $[12,13]$.

Sensitive and selective detection of biomarkers in real samples is a great challenge in the field of electrochemical assays, because it requires the surpassing of natural interferences, such as lipids, sugars, and proteins (or their metabolism) commonly found in plasma, serum, cerebrospinal fluid, saliva, and urine, that cause unwanted non-specific interactions [14]. These nonspecific interactions are generally hydrophobic, electrostatic, 


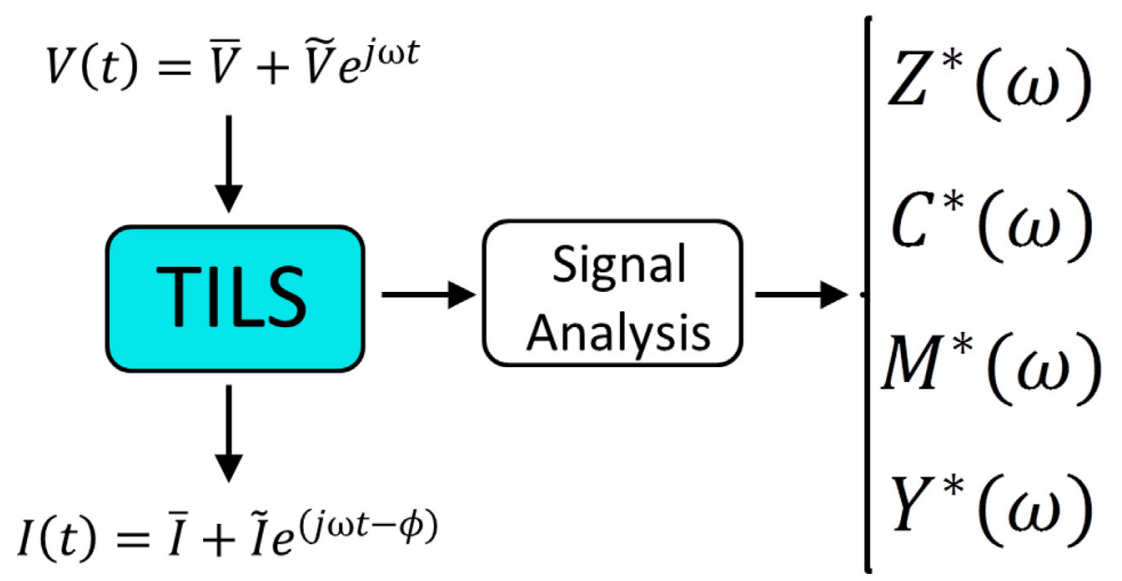

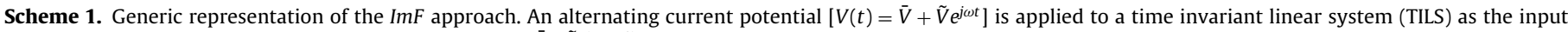

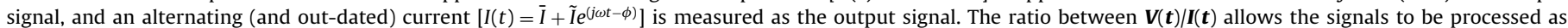

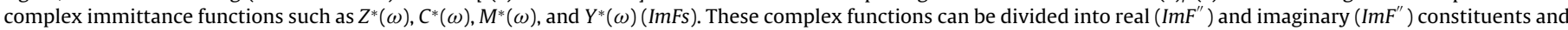

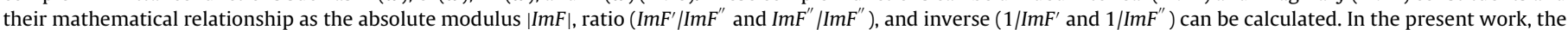

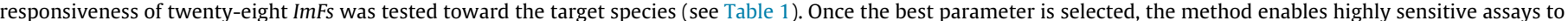
be performed quickly at an independent single frequency.

or hydrogen-bonding in nature. Consequently, hydrophilic and highly hydrated films, developed using PEGylation chemistry, have been successfully used to obtain low-fouling interfaces for electrochemical assays [15-17]. SAMs composed of PEGylated thiols possess low-fouling properties and have produced reliable results for detecting biomarkers in serum media [16]. Therefore, herein we have used a mixed SAM composed of 11-ferrocenylundecanethiol $(11 \mathrm{FcC})$ and polyethylene glycol (PEG) containing the thiol HS- $\left(\mathrm{CH}_{2}\right)_{11}-(\mathrm{EG})_{3}-\mathrm{OCH}_{2}-\mathrm{COOH}$ (PEG thiol) for detecting human prostatic acid phosphatase ( $h \mathrm{PAP}$ ) in phosphate-buffered saline PBS and blood human serum. hPAP is a major phosphatase enzyme composed of differentiated prostate epithelial cells $[18,19]$ which is biosynthesized by the columnar secretory epithelia of the prostate gland [20] and, in normal healthy individuals, its plasma levels are on the order of $1-3 \mathrm{ng} \mathrm{mL}^{-1}(10-30 \mathrm{pM})$. However, elevated circulating levels of $h$ PAP have long been considered as an additional biomarker for prostate cancer [21-23]. In addition, $h P A P$ has been demonstrated to be a good prognostic marker for patients with aggressive diseases undergoing local therapy that are at high risk of distant relapse. Therefore, developing quick, sensitive, cheap, and high-throughput new methods for monitoring hPAP is clearly important for both diagnostic and therapeutic controls. Electrochemical techniques are potentially suitable for achieving such demands; however, hPAP detection by electric/electrochemical techniques has been poorly explored so far and, to the best of our knowledge, $h$ PAP detection has only tested using amperometric [24] and ECS [4] approaches. Thus, herein we propose a new electrochemical approach for detecting $h$ PAP using immittance analysis combined with a redox-SAM configuration in which the complex functions $\left(Z^{*}, C^{*}, Y^{*}\right.$ and $\left.M^{*}\right)$ are quantitatively analysed across a frequency range spanning seven orders of magnitude (from $0.01 \mathrm{~Hz}$ to $1.0 \mathrm{MHz}$ ). Based on the most sensitive response with the lowest background noise, the optimum parameters, frequencies, and frequency range are then selected for obtaining analytical curves with a high diagnostic utility for $h$ PAP detection in human blood serum.

\section{Material and methods}

\subsection{Chemical reagents}

Ethanolamine (98\%), 1-ethyl-3-(3-dimethylaminopropyl) carbodiimide (EDC), $N$-hydroxysuccinimde (NHS), hPAP antibody, $h P A P$, and 11-ferrocenyl-undecanethiol (11-FcC) were purchased from Sigma Aldrich, while polyethylene glycol (PEG) containing thiol HS- $\left(\mathrm{CH}_{2}\right)_{11}-(\mathrm{EG})_{3}-\mathrm{OCH}_{2}-\mathrm{COOH}$ (PEG thiol) was purchased from Prochimia Surfaces. Phosphate buffered saline (PBS, pH 7.4) solution was prepared with a composition of $8 \mathrm{~g} \mathrm{~L}^{-1} \mathrm{NaCl}, 0.2 \mathrm{~g} \mathrm{~L}^{-1}$ $\mathrm{KH}_{2} \mathrm{PO}_{4}, 1.15 \mathrm{gL}^{-1} \mathrm{NaH}_{2} \mathrm{PO}_{4} \cdot 12 \mathrm{H}_{2} \mathrm{O}, 0.2 \mathrm{gL}^{-1} \mathrm{KCl}$, and $0.2 \mathrm{gL}^{-1}$ $\mathrm{NaNO}_{3}$ and all chemicals were of analytical grade. Deionized water (18.2 $\mathrm{M} \Omega \mathrm{cm}^{-1}$, Synergy Ultrapure water system EMD Millipore) was used throughout. All other chemicals used in the experiments described in this work were of analytical grade.

\subsection{Apparatus}

Cyclic voltammetry (CV) and electrochemical impedance spectroscopic (EIS) experiments were conducted with an Autolab Potentiostat equipped with an FRA module and using the following three-electrode setup: conventional gold disk working electrodes of $2.0 \mathrm{~mm}$ diameter (Metrohm), platinum wire counter electrode, and silver/silver chloride $(\mathrm{Ag} \mid \mathrm{AgCl}$, filled with $3.0 \mathrm{M} \mathrm{KCl})$ reference electrode. All EIS responses were recorded over a wide range of frequencies from $0.01 \mathrm{~Hz}$ to $1.0 \mathrm{MHz}$ (on a logarithmic scale). The error bars are the standard deviations of three successive measurements with the same electrode, with each experiment repeated five times.

\subsection{Fabrication of bio-recognition layer and sensor testing}

Gold disk electrodes were mechanically polished (with aluminium oxide pads of three successive sizes: $1 \mu \mathrm{m}, 0.3 \mu \mathrm{m}$, and $0.05 \mu \mathrm{m}$ ) to prepare flat, mirror-like surfaces. This was followed by rinsing and ultra-sonic washing with ultrapure water and absolute ethanol. The electrode surface was then electrochemically polished in a $0.5 \mathrm{M} \mathrm{KOH}$ aqueous solution by applying negative CV scans from -1.7 to $-0.7 \mathrm{~V}$ (200 cycles) to remove any chemically or physically adsorbed molecules. The gold oxide was then removed using chemical reduction by incubation with absolute EtOH at ambient temperature under stirring for $20 \mathrm{~min}$. A series of wider range scans, from -0.1 to $1.4 \mathrm{~V}$, were then conducted in $0.5 \mathrm{M} \mathrm{H}_{2} \mathrm{SO}_{4}$ at a scan rate of $0.1 \mathrm{~V} \mathrm{~s}^{-1}$, until the height and shape of the anodic and cathodic peaks were invariant. Electrical stripping was then carried out by running 10 successive CV scans from $0.75 \mathrm{~V}$ to $0.2 \mathrm{~V}$ in a $0.5 \mathrm{M} \mathrm{H}_{2} \mathrm{SO}_{4}$ aqueous solution at scan rate of $0.1 \mathrm{~V} \mathrm{~s}^{-1}$ to remove the gold oxide. The reduction peak of the gold oxide layer formed in the anodic scan was used to calculate the real electroactive area $\left(A_{R}\right)$ of the electrode. The electrode was only 

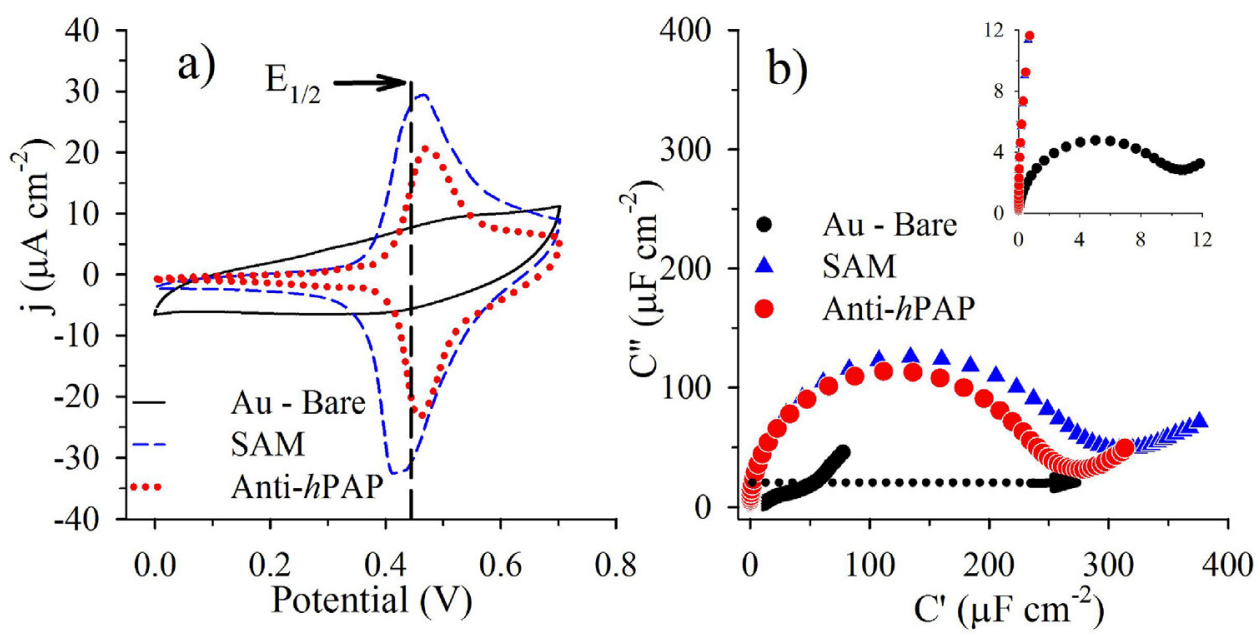

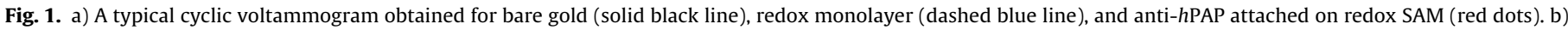

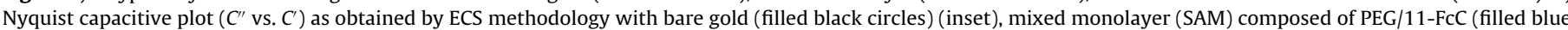

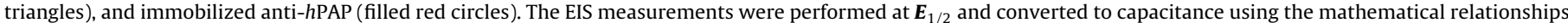
$\boldsymbol{C}^{\prime}=1 / \boldsymbol{j} \omega \boldsymbol{Z}^{\prime \prime}$ and $\boldsymbol{C}^{\prime \prime}=1 / \boldsymbol{j} \omega \boldsymbol{Z}^{\prime}$.

used for further experiments if the roughness factor $\left(R_{F}=A_{R} / A_{G}\right.$, where $A_{G}$ is the geometric area) was between 1.1 and 1.4 ; otherwise, the cleaning procedure was repeated. Thus, the bare gold electrodes were characterized by CV and EIS and the electrochemical polishing was repeated again before immersing the electrodes in a mixed ethanol (HPLC grade) solution of $0.02 \mathrm{mM}$ PEG thiol and $1.98 \mathrm{mM} 11-\mathrm{FcC}(1: 99 \%)$ for $16 \mathrm{~h}$. The electrodes were then rinsed with absolute ethanol to remove any unbound molecules and dried under nitrogen flow prior to CV and EIS measurements. After SAM characterization, the electrodes were immersed in a solution of 1-ethyl-3-(3-dimethylaminopropyl) carbodiimide (EDC) (0.4 M) and $\mathrm{N}$-hydroxysuccinimide (NHS) $(0.1 \mathrm{M})$ in ultrapure water for 40 min to activate the terminal carboxyl groups of the PEGylate. The electrodes were then incubated in $1 \mu \mathrm{M}$ of anti- $h$ PAP antibody in PBS solution for $1 \mathrm{~h}$ at room temperature $[25,26]$ and CV and EIS assays were performed again to certify the antibody immobilization. Finally, the remaining activated carboxylic groups were deactivated by incubation in a $1 \mathrm{M}$ ethanolamine solution $(\mathrm{pH}$ $\sim 8.5$ ). The bio-recognition layers were then immersed in a $0.15 \mathrm{M}$ phosphate buffer solution ( $\mathrm{pH} 7.3$ ) containing specific quantities of $h$ PAP ranging from $20 \mathrm{pM}$ to $5000 \mathrm{pM}$. The incubation time was $30 \mathrm{~min}$ for each concentration and the electrodes were washed with PBS solution before electrochemical analysis. When evaluating the interfacial specificity fetuin-A was used as control since it is a glycoprotein formed by liver cells that is secreted into the serum at high concentrations $[27,28]$. The specificity was evaluated by monitoring the $h \mathrm{PAP}$ in the presence of the matrix components, in this case by adding $h$ PAP in human blood serum (instead of PBS). The linear range in this configuration was found to be $50 \mathrm{pM}$ to $10^{4} \mathrm{pM}$.

\subsection{Electrochemical measurements and data processing}

Cyclic voltammetry and impedance-derived capacitance analyses were carried out at all stages of the biosensor fabrication/modification. From CV analyses the half-wave potential $\left(E_{1 / 2}\right)$ was obtained and then used to conduct the impedance-derived capacitance analysis (Fig. 1). All electrochemical measurements were recorded in a supporting electrolyte of $20 \mathrm{mM} \mathrm{TBAClO}_{4}$ (tetrabutylammonium perchlorate) dissolved in acetonitrile and $\mathrm{H}_{2} \mathrm{O}$ $(20: 80)$ vol\% without any redox probe (amplifier) present in the electrolyte. CV scans were performed at a scan rate of $100 \mathrm{mV} \mathrm{s}^{-1}$ between $0.0 \mathrm{~V}$ and $0.7 \mathrm{~V}$ relative to $\mathrm{Ag} \mid \mathrm{AgCl}$, while EIS measurements were conducted over a frequency range of $0.01 \mathrm{~Hz}$ to $1 \mathrm{MHz}$
Table 1

All $\operatorname{Im} F$ parameters evaluated in the methodology applied to $h$ PAP detection. This ImF library was previously published in Refs. [12] and [13].

\begin{tabular}{llll}
\hline$I m F$ & & & \\
\hline$Z^{*}$ & $C^{*}$ & $M^{*}$ & $Y^{*}$ \\
$Z^{\prime}$ & $C^{\prime}$ & $M^{\prime}$ & $Y^{\prime}$ \\
$Z^{\prime \prime}$ & $C^{\prime \prime}$ & $M^{\prime \prime}$ & $Y^{\prime \prime}$ \\
$|Z|$ & $|C|$ & $|M|$ & $|Y|$ \\
$Z^{\prime} \mid Z^{\prime \prime}$ & $C^{\prime} / C^{\prime \prime}$ & $M^{\prime} / M^{\prime \prime}$ & $Y^{\prime} / Y^{\prime \prime}$ \\
$Z^{\prime \prime} \mid Z^{\prime}$ & $C^{\prime \prime} / C^{\prime}$ & $M^{\prime \prime} \mid M^{\prime}$ & $Y^{\prime \prime} / Y^{\prime}$ \\
$1 / Z^{\prime}$ & $1 / C^{\prime}$ & $1 / M^{\prime}$ & $1 / Y^{\prime}$ \\
$1 / Z^{\prime \prime}$ & $1 / C^{\prime \prime}$ & $1 / M^{\prime \prime}$ & $1 / Y^{\prime \prime}$ \\
\hline
\end{tabular}

with an amplitude of $10 \mathrm{mV}$ peak to peak at $E_{1 / 2}$. The parameters collected from EIS analysis such as voltage $\left(V(t)=\bar{V}+\tilde{V} e^{j \omega t}\right)$ and current $\left(I(t)=\bar{I}+\tilde{I} e^{(j \omega t-\phi)}\right)$, which are the input and output signal, respectively, were used to calculate the immittance function parameters, ImFs; firstly the impedance was calculated $\left(Z^{*}=\right.$ $V(t) / I(t))$ followed by calculation of the capacitance $\left(C^{*}=1 / j \omega Z^{*}\right)$, modulus $\left(M^{*}=\omega Z^{*}\right)$, and admittance $\left(Y^{*}=j \omega C^{*}\right)$, where $j=\sqrt{-1}$, $\omega=2 \pi f$, and $\phi$ is the phase angle. The bar and $\sim$ over the potential or current indicates steady state and amplitude, respectively. Furthermore, all ImFs were individually divided into constituent real $\left(I m F^{\prime}\right)$ and imaginary $\left(I m F^{\prime \prime}\right)$ components (since $\operatorname{ImFs}$ are complex functions). Using the real and imaginary components we additionally calculated sub-parameters, such as the absolute value $(|\operatorname{ImF}|)$, with their ratios $\left(I m F^{\prime} / \operatorname{ImF^{\prime \prime }}\right)$ and inverted ratios $\left(\operatorname{Im} F^{\prime \prime} / \operatorname{Im} F^{\prime}\right)$ (totalizing 28 parameters as described in Table 1). The ImF parameters (and sub-parameters) were normalized using the percentage relative response (RR\%), which is calculated as $R R_{n}^{f}(\%)=\left(\left(R_{n}^{f}-R_{0}^{f}\right) / R_{0}^{f}\right) * 100$, where $R_{0}^{f}$ represents the blank (in the absence of analyte) and $R_{n}^{f}$ represents the $\operatorname{Im} F$ value after interaction with a specific target concentration $(n)$ at the same frequency $f$.

\section{Results and discussion}

To guarantee the efficacy and applicability of any given redox-monolayer-based biosensor, the bio-recognition-layer construction must be characterized step-by-step in order to certify the SAM formation and antibody immobilization. Thus, CV and EIS

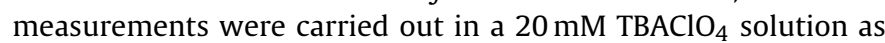




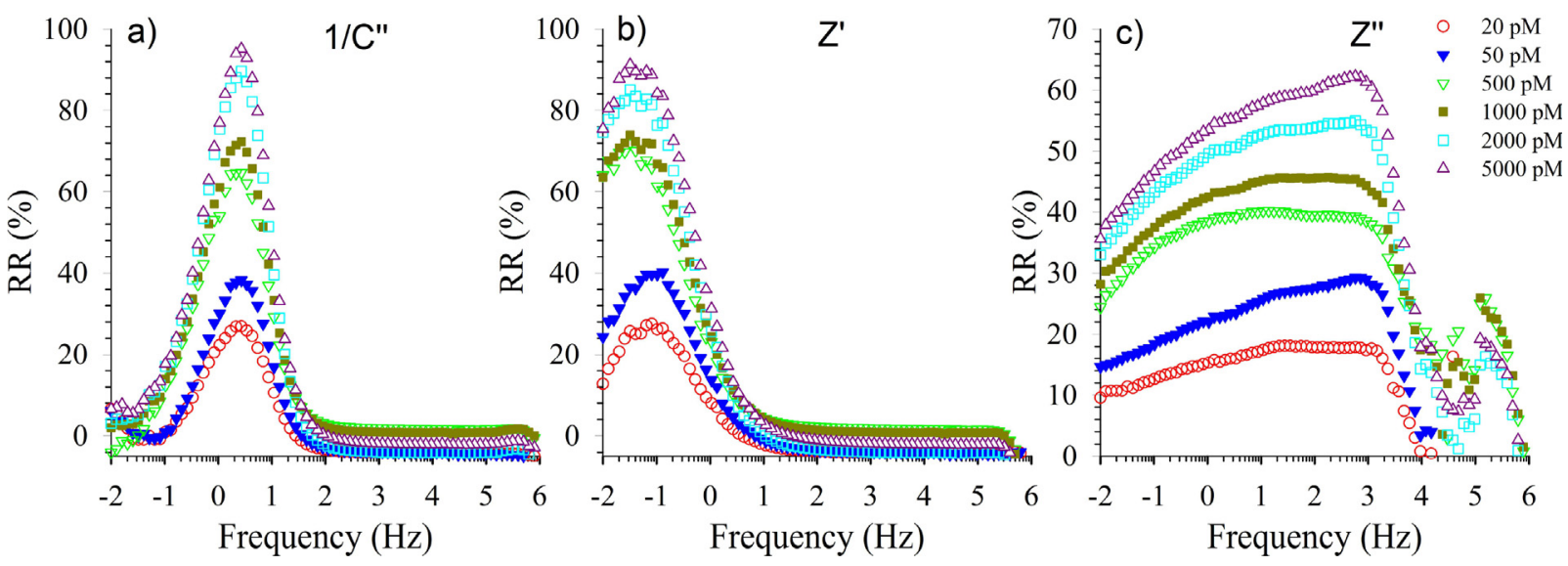

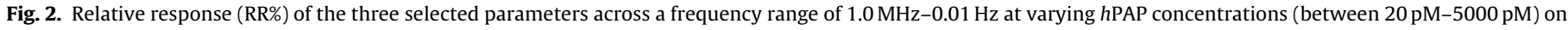

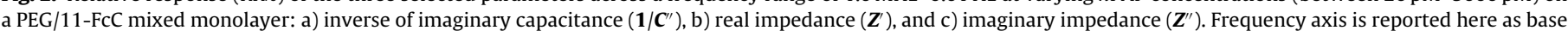
ten exponents.

detailed in the Materials and Methods (2.4) Section. As expected, the bare gold surface showed only non-faradic capacitive behaviour as evident in the CV plot in Fig. 1a and the Nyquist capacitive plot in Fig. 1b (inset), where the capacitance data were derived from the impedimetric analysis with the real and imaginary components defined as $C^{\prime}=1 / j \omega Z^{\prime \prime}$ and $C^{\prime \prime}=1 / j \omega Z^{\prime}$, respectively.

After formation of the PEG/11FCC SAM, the faradaic activity of the confined redox probe $(11-\mathrm{FcC})$ is evident from the appearance of anodic and cathodic peaks with intensities of $-31.0 \mu \mathrm{Acm}^{-2}$ and $29.5 \mu \mathrm{Acm}^{-2}$, respectively, around $0.45 \mathrm{~V}$ (Fig. 1a). The huge increase in capacitance from $9.0 \mu \mathrm{F} \mathrm{cm}^{-2}$ for bare gold to $316 \mu \mathrm{F} \mathrm{cm}^{-2}$ after SAM formation, as estimated from the size of the semicircles in Fig. 1b, is also related to the faradaic activity of 11FcC [13]. Both the CV peaks and "supercapacitance" value depend on the density of the immobilized redox probe, or more precisely on the density of accessible redox states as given by $g(\bar{\mu})=d N / d \bar{\mu}$, where $\bar{\mu}$ is the electrochemical potential of the junction and $N$ is the concentration of charged (positive or negative) redox centres within the interface $[5,8-11,29]$. Incubation in the anti-hPAP solution clearly caused a reduction in both the cathodic $(p c)$ and anodic ( $p a$ ) peak currents, with $p c$ reduced from $29.5 \pm 3.4 \mu \mathrm{Acm}^{-2}$ to $20.1 \pm 1.6 \mu \mathrm{Acm}^{-2}$, as shown in Fig. $1 \mathrm{a}$, and the capacitance reduced to $270 \pm 4 \mu \mathrm{F} \mathrm{cm}^{-2}$ (from $316 \pm 20 \mu \mathrm{F} \mathrm{cm}^{-2}$ ). This indicates that the anti-hPAP is immobilization on the SAM.

Having confirmed the redox-monolayer formation and the attachment of the receptor, the electrode surface was tested against different concentrations of the target species in PBS in order to evaluate the applicability of the bio-recognition layer and, thus, optimize the best frequency and $\operatorname{Im} F$ parameters. The raw electrochemical spectroscopic data (from $1 \mathrm{MHz}$ to $0.01 \mathrm{~Hz}$ at $E_{1 / 2}$ ) was mathematically converted to the $\operatorname{Im} F$ parameters $\left(Z^{*}, C^{*}, Y^{*}\right.$ and $\left.M^{*}\right)$ and then normalized by calculating the percentage relative response, as described in the Materials and Methods section. It should be noted that the responsiveness was evaluated for each $\operatorname{Im} F$ parameter at every frequency studied in the raw data. However, only those parameters showing acceptable linearity $\left(\mathrm{r}^{2}>0.98\right)$ at a given frequency were used to obtain the analytical curves, while responses with $\mathrm{r}^{2}<0.98$ were ignored. The optimal frequency for each $\operatorname{ImF}$ was then chosen by considering the highest sensitivity (S) (slope of the analytical curve) obtained. These analytical curves were then used to calculate the LoD from the standard deviation (SD) of the blank response and the slope of the analytical curve $[(3.3 \times \mathrm{SD}) / \mathrm{S})$, where $\mathrm{SD}$ is the standard deviation of the blank measurements $(n=10)$ and $S$ is the slope of the linear response]. The performance and optimal frequency of each $\operatorname{Im} F$ parameter were compared, with the aim of determining the most efficient $I m F$

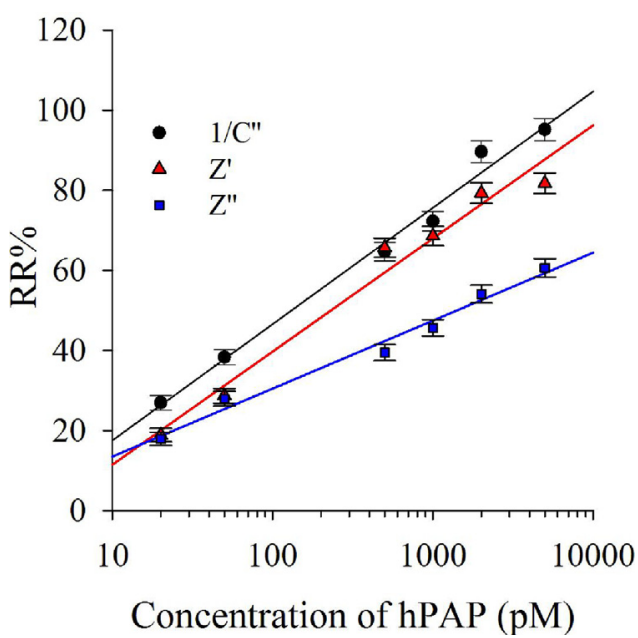

Fig. 3. Comparative function responses to $h P A P$ detection using a PEG/11FcCmodified gold electrode for the most responsive parameters $1 / \boldsymbol{C}^{\prime \prime}, \boldsymbol{Z}^{\prime}$, and $\boldsymbol{Z}^{\prime \prime}$. The error bars represent the standard deviation of three measurements for each concentration using the same electrode. The relative standard deviations (RSD) were between $6.7 \%$ and $2.9 \%$ for measurements for $\mathbf{1} / \mathbf{C}^{\prime \prime}$, between $9.3 \%$ and $3.7 \%$ for $\boldsymbol{Z}$, and between $9.0 \%$ and $3.1 \%$ for $\boldsymbol{Z}^{\prime \prime}$.

parameter. The $1 / C^{\prime \prime}, Z^{\prime}$, and $Z^{\prime \prime}$ immittance function parameters showed the best analytical performance among all ImFs studied here (as demonstrated in Table 2).

Fig. 2 shows the spectral response in RR\% for $1 / C^{\prime \prime}, Z^{\prime}$, and $Z^{\prime \prime}$ over the frequency range studied and in the presence of a specific concentration of the target species. Clearly, the variation in RR\% across the frequency range as a function of target species (hPAP) concentration is different for each of the parameters. In addition, the optimal frequency was different for each $\operatorname{ImF}$. Significant responses were found between $8 \mathrm{~Hz}$ and $0.8 \mathrm{~Hz}$ for $1 / C^{\prime \prime}$ (Fig. 2a) and below $0.3 \mathrm{~Hz}$ for $Z^{\prime}$ (Fig. 2b). On the other hand, $Z^{\prime \prime}$ showed a significant response at the high frequencies spanning from $1900 \mathrm{~Hz}$ to $1.0 \mathrm{~Hz}$ (Fig. 2c). The entire data processing, from its conversion from original electrical transfer functions (impedimetric data), calculation of linearity, sensitivity, and LoDs, was performed using a homewritten MATLAB R2014a algorithm, which renders it possible to perform the entire process in a few seconds.

A linear range was found between $20 \mathrm{pM}$ to $5000 \mathrm{pM}$ for all three parameters. The optimal frequency for $1 / C^{\prime \prime}$ was $2.69 \mathrm{~Hz}$, with a sensitivity of $29.2 \%$ per decade of concentration range (\% conc ${ }^{-1}$ ) and an LoD of $4.1 \pm 1.3 \mathrm{pM}$. The best frequency for $Z^{\prime}$ was $0.02 \mathrm{~Hz}$, 
Table 2

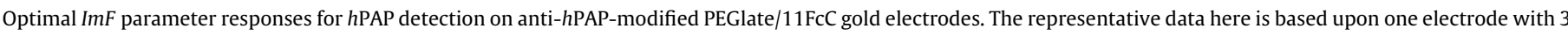

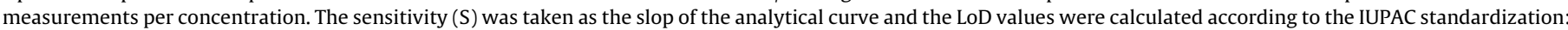
$\mathrm{LoD}=(3.3 \times \mathrm{SD}) / \mathrm{S})$, where $\mathrm{SD}$ is the standard deviation of the blank measurements (around 10$)$ and $\mathrm{S}$ is the slope of the linear response.

\begin{tabular}{|c|c|c|c|c|}
\hline \multicolumn{5}{|c|}{$h$ PAP detection } \\
\hline $\operatorname{Im} F$ & Optimized Frequency (Hz) & $\mathrm{r}^{2}$ & Sensitivity $\left(\%\right.$ conc $\left.^{-1}\right)$ & LoD $(\mathrm{pM})$ \\
\hline$Z^{\prime}$ & 0.0201 & 0.980 & 28.3 & $6.8 \pm 2.1$ \\
\hline$Z^{\prime \prime}$ & 179.1 & 0.981 & 17.3 & $4.0 \pm 0.9$ \\
\hline $1 / C^{\prime \prime}$ & 2.6939 & 0.988 & 29.2 & $4.1 \pm 1.3$ \\
\hline
\end{tabular}
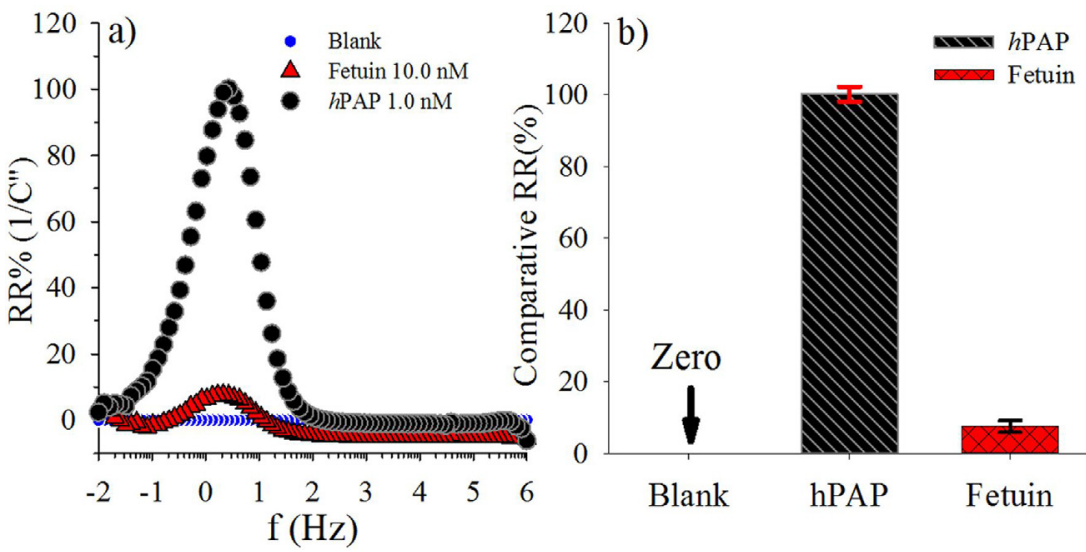

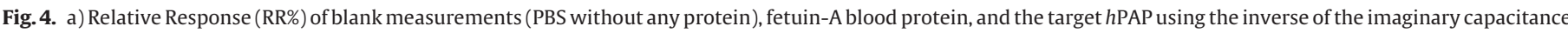

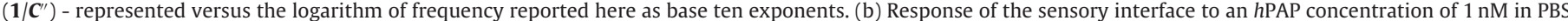

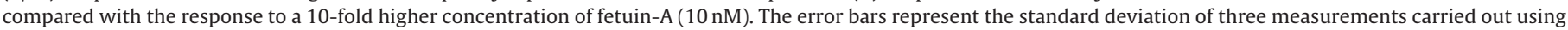
a single functionalized electrode.
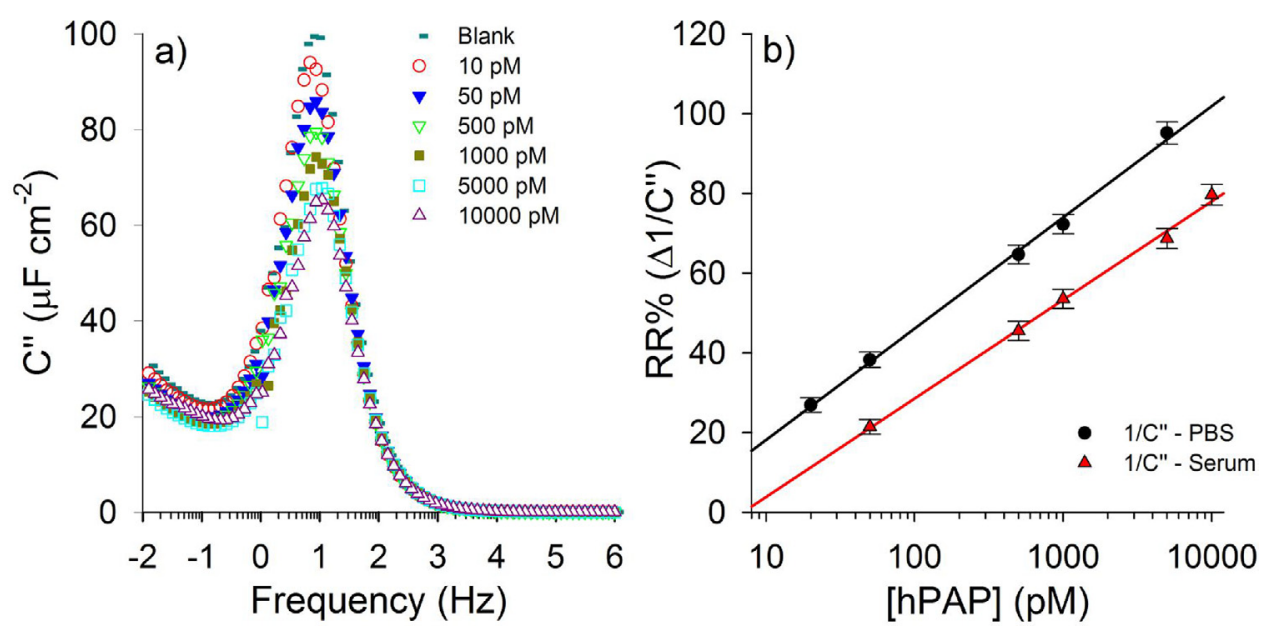

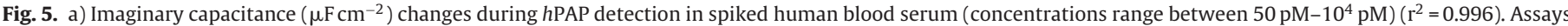

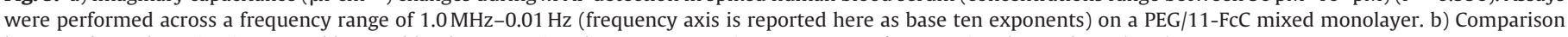
between $h$ PAP detection in PBS and human blood serum using the most responsive parameter, $\mathbf{1} / \mathbf{C}^{\prime \prime}$, as a signal transducer (RR\%).

with a sensitivity of $28.3 \%$ conc $^{-1}$ and an LoD of $6.8 \pm 2.1 \mathrm{pM}$, while the optimal frequency for $Z^{\prime \prime}$ was $179.1 \mathrm{~Hz}$, with a sensitivity of $17.3 \%$ conc $^{-1}$ and an LoD of $4.0 \pm 0.9 \mathrm{pM}$ (the main values of merit are summarized in Table 2). A comparison of the analytical curves obtained for $1 / C^{\prime \prime}, Z^{\prime}$ and $Z^{\prime \prime}$ is shown in Fig. 3, with the error bars showing the standard deviation among three measurements. These calculated LoD values (less than $10.0 \mathrm{pM}$ ) and linear ranges (20 pM-5000 pM) compare favourably with ELISA (Enzyme-Linked Immunosorbent Assay) [30] and other electrochemical methods [4]. Additionally, the methodology proposed herein is applicable over a clinically useful range, given that healthy plasma levels of $h$ PAP are on the order of $10 \mathrm{pM}$ to $30 \mathrm{pM}$ and elevated levels around $100 \mathrm{pM}$ are indicative of prostate cancer [30]. The repeatability and reproducibility of this approach was tested by measuring the target species at a concentration of $100 \mathrm{pM}$, which is a level indicative of prostate cancer [30]. Using $1 / C^{\prime \prime}$ at the optimum frequency, the relative standard deviation (RSD) was $3.6 \%$ and $8.9 \%$ for intra-day and inter-day analyses (over 6 runs), respectively. Furthermore, it is possible to perform the assay and data analysis in less than $1 \mathrm{~min}$ by using any of the optimal frequencies or even by limiting the sampling frequency range to between $200 \mathrm{~Hz}$ and $1.0 \mathrm{~Hz}$ (where the maximal sensitivities for $1 / C^{\prime \prime}$ and $Z^{\prime \prime}$ were found). To the best of our knowledge, this is the fastest method for detecting hPAP reported in the literature.

We also evaluated the interfacial specificity by measuring the interaction of a freshly prepared modified electrode (with PEG/11- 
FcC and anti- $h$ PAP) with fetuin-A, as described in the Materials and Methods (2.3). As shown in Fig. 4, we compared the optimum response of $1 / C^{\prime \prime}$ toward $h \mathrm{PAP}(2.69 \mathrm{~Hz})$, at a concentration of $1 \mathrm{nM}$, with that toward a fetuin-A concentration of $10 \mathrm{nM}$ (10fold higher); we found a variation of $100 \pm 2 \%$ for the biomarker and a variation of $7.4 \pm 1.7 \%$ for the fetuin-A (approximately 13.5 fold lower than that for $h \mathrm{PAP}$ ). This confirms the specificity of the method toward $h$ PAP and clearly shows that the response toward interfering species is negligible in comparison with the targetspecies response.

Considering the consistent results obtained in PBS and the specificity confirmed by the negative control test using fetuin-A, we prepared $\operatorname{Im} F$ assays using $1 / C^{\prime \prime}$ as a signal transducer to detect $h \mathrm{PAP}$ in human blood serum. A linear response was exhibited for target concentrations ranging from $50 \mathrm{pM}$ to $10^{4} \mathrm{pM}\left(\mathrm{r}^{2}=0.996\right)$ at $5.4 \mathrm{~Hz}$ (optimum frequency). The Bode plot in Fig. 5a shows the spectral response of $C^{\prime \prime}\left(\mu \mathrm{F} \mathrm{cm}^{-2}\right)$ for each $h \mathrm{PAP}$ concentration, while Fig. 5b compares the linear response using $1 / C^{\prime \prime}$ (RR\%) for $h P A P$ detection in PBS and serum. The sensitivity in serum was $24.7 \%$ conc $^{-1}$, a value similar to the $29.2 \%$ conc $^{-1}$ achieved in PBS, while the LoD for the assays in serum was $11.2 \pm 2.6 \mathrm{pM}$, which is practically the same order as that found in PBS $(4.1 \pm 1.3 \mathrm{pM})$ and comparably better than that achieved using ELISA methods [30]. The increase in the LoD compared with the LoD values obtained in PBS was expected due to the well-known serum matrix effects, though the values are still clinically useful when conducting measurements directly in serum.

\section{Conclusion}

The combination of a low-fouling and redox-nanostructured surface with an immittance-function data-processing analytical method provides a new and efficient label-free methodology for detecting $h \mathrm{PAP}$ without the need to add any redox probe agents to the biological sample. This method is a potential point-of-care approach. This study has shown that, provided the optimal frequency range is known, it is possible to perform assays and data analysis faster than any other method developed for this target species and, additionally, to achieve high assay sensitivity and LoD values that are equivalent or better than those obtained using ELISA or other alternative and traditional electrochemical methods.

\section{Acknowledgements}

This work was supported by the São Paulo state researchfunding agency (FAPESP) and CNPq. Flávio Bedatty acknowledges the FAPESP for his Post Doc scholarship and grants. We also acknowledge Amol Patil who developed the MATLAB R2014a algorithm code.

\section{References}

[1] F.C.B. Fernandes, A. Santos, D.C. Martins, M.S. Góes, P.R. Bueno, Comparing label free electrochemical impedimetric and capacitive biosensing architectures, Biosens. Bioelectron. 57 (2014) 96-102.

[2] A. Santos, F.C. Carvalho, M.-C. Roque-Barreira, P.R. Bueno, Impedance-derived electrochemical capacitance spectroscopy for the evaluation of lectin-glycoprotein binding affinity, Biosens. Bioelectron. 62 (2014) 102-105.

[3] F.C.B. Fernandes, M.S. Góes, J.J. Davis, P.R. Bueno, Label free redox capacitive biosensing, Biosens. Bioelectron. 50 (2013) 437-440.

[4] J. Lehr, F.C.B. Fernandes, P.R. Bueno, J.J. Davis, Label-free capacitive diagnostics: exploiting local redox probe state occupancy, Anal. Chem. 86 (2014) 2559-2564.

[5] P.R. Bueno, G. Mizzon, J.J. Davis, Capacitance spectroscopy: a versatile approach to resolving the redox density of states and kinetics in redox-active self-assembled monolayers, J. Phys. Chem. B 116 (2012) 8822-8829.

[6] J. Cecchetto, F.C.B. Fernandes, R. Lopes, P.R. Bueno, The capacitive sensing of NS1 Flavivirus biomarker, Biosens. Bioelectron. 87 (2017) 949-956
[7] A. Santos, J.P. Piccoli, N.A. Santos-Filho, E.M. Cilli, P.R. Bueno, Redox-tagged peptide for capacitive diagnostic assays, Biosens. Bioelectron. 68 (2015) 281-287.

[8] P.R. Bueno, G.T. Feliciano, J.J. Davis, Capacitance spectroscopy and density functional theory, Phys. Chem. Chem. Phys. 17 (2015) 9375-9382.

[9] P.R. Bueno, F. Fabregat-Santiago, J.J. Davis, Elucidating capacitance and resistance terms in confined electroactive molecular layers, Anal. Chem. 85 (2013) 411-417.

[10] P.R. Bueno, J.J. Davis, Elucidating redox-level dispersion and local dielectric effects within electroactive molecular films, Anal. Chem. 86 (2014) 1997-2004.

[11] P.R. Bueno, J.J. Davis, Measuring quantum capacitance in energetically addressable molecular layers, Anal. Chem. 86 (2014) 1337-1341.

[12] A.V. Patil, F.C. Bedatty Fernandes, P.R. Bueno, J.J. Davis, Immittance electroanalysis in diagnostics, Anal. Chem. (2014).

[13] F.C. Bedatty Fernandes, A.V. Patil, P.R. Bueno, J.J. Davis, Optimized diagnostic assays based on redox tagged bioreceptive interfaces, Anal. Chem. 87 (2015) 12137-12144.

[14] Q. Xu, H. Cheng, J. Lehr, A.V. Patil, J.J. Davis, Graphene oxide interfaces in serum based autoantibody quantification, Anal. Chem. 87 (2015) 346-350.

[15] X. Luo, Q. Xu, T. James, J.J. Davis, Redox and label-free array detection of protein markers in human serum, Anal. Chem. 86 (2014) 5553-5558.

[16] T. Bryan, X. Luo, L. Forsgren, L.A. Morozova-Roche, J.J. Davis, The robust electrochemical detection of a Parkinson's disease marker in whole blood sera, Chem. Sci. 3 (2012) 3468-3473.

[17] Y. Zheng, T. Hu, C. Chen, F. Yang, X. Yang, An anti-fouling aptasensor for detection of thrombin by dual polarization interferometry, Chem. Commun. 51 (2015) 5645-5648.

[18] O. Bodansky, Reflections on biochemical aspects of human cancer, Cancer 33 (1974) 364-370.

[19] N. Azumi, S.T. Traweek, H. Battifora, Prostatic acid phosphatase in carcinoid tumors. Immunohistochemical and immunoblot studies, Am. J. Surg. Pathol, 15 (1991) 785-790

[20] P. Vihko, Characterization of the principal human prostatic acid phosphatase isoenzyme, purified by affinity chromatography and isoelectric focusing. Part II, Clin. Chem. 24 (1978) 1783-1787.

[21] F.R. Ahmann, R.B. Schifman, Prospective comparison between serum monoclonal prostate specific antigen and acid phosphatase measurements in metastatic prostatic cancer, J. Urol. 137 (1987) 431-434

[22] J.M. Robles, A.R. Morell, J.P. Redorta, J.A.d.T. Mateos, A.S. Roselló, Clinical behavior of prostatic specific antigen and prostatic acid phosphatase: a comparative study, Eur. Urol. 14 (1988) 360-366.

[23] S. Muniyan, N. Chaturvedi, J. Dwyer, C. LaGrange, W. Chaney, M.-F. Lin, Human prostatic acid phosphatase: structure, function and regulation, Int. J. Mol. Sci. 14 (2013) 10438-10464

[24] J. Castañón-Fernández, M.T. Fernández-Abedul, A. Costa-García, Determination of acid phosphatase activity in a double injection flow system with electrochemical detection, Anal. Chim. Acta 406 (2000) 225-232.

[25] T. Bryan, X. Luo, P.R. Bueno, J.J. Davis, An optimised electrochemical biosensor for the label-free detection of C-reactive protein in blood, Biosens. Bioelectron. 39 (2013) 94-98.

[26] M. Xu, X. Luo, J.J. Davis, The label free picomolar detection of insulin in blood serum, Biosens. Bioelectron. 39 (2013) 21-25.

[27] K.O. Pedersen, Fetuin, a new globulin isolated from serum, Nature 154 (1944) 575.

[28] M. Singh, P.K. Sharma, V.K. Garg, S.C. Mondal, A.K. Singh, N. Kumar, Role of fetuin-A in atherosclerosis associated with diabetic patients, J. Pharm. Pharmacol. 64 (2012) 1703-1708.

[29] D.A. Miranda, P.R. Bueno, Density functional theory and an experimentally-designed energy functional of electron density, Phys. Chem. Chem. Phys. 18 (2016) 25984-25992

[30] M.P.v. Dieijen-Visser, K.P.J. Delaere, A.H.J. Gijzen, P.J. Brombacher, A comparative study on the diagnostic value of prostatic acid phosphatase (PAP) and prostatic specific antigen (PSA) in patients with carcinoma of the prostate gland, Clin. Chim. Acta 174 (1988) 131-140.

\section{Biographies}

Professor Paulo R. Bueno is coordinator of the Nanobionics research group (www.nanobionics.pro.br) at São Paulo State University, UNESP. He published over 170 papers related to the applications or development of mainly electric and electrochemistry time-dependent (including gravimetric and optical impedance) spectroscopic methods (h index 30, >3500 citations and with an average citation above 20 per article). Nowadays, his main scientific interest is focused on the fundamental aspects and technological applications involving electron charge transfer and transport as well as the charge storage occurring in nanoscale (molecular) and supra-molecular (bio-molecular) structures. Under his guidance Nanobionics group develops synthetic structures and surfaces that mimics molecular and biological structures to be used, for instance, applied in highly-relevant medical diagnostic devices. The Nanobionics group was responsible for the development of a totally new label-free diagnostic assay and electro analytical spectroscopic methodology supported on the use of the electrochemistry capacitance, a capacitance proportional to the electrochemical density of states (EDOS) that intrinsically exist in mesoscopic structures comprised of redox-active molecules attached over metal- 
lic probes, particularly named as Electrochemistry Capacitance Spectroscopy (ECS). The ECS-derived assays are able to detect target protein at femtomolar levels allowing the establishment of an early detection assay platforms for clinically important targets and that are better than those currently in use and are potentially cheap and useful in multiplexed and bedside like-diagnostic technologies. Other relevant aspects of his groups' activities are the synthesis of self-assembled redox-tagged peptides and its applications in redox capacitive biosensors. The advantages of constructing a peptide-based receptive surface containing electrochemical tags is that peptides can be easily manipulated and optimized to be applied directly in complex biological environments avoiding fouling. Additionally antibodies/antigens can be substituted by peptide aptamers with equivalent affinity and specificity for the clinical target proteins. In the molecular design and surface engineering context, the Nanobionics group is supported by computational chemistry expertise and tools such as molecular dynamics (MD) and quantum mechanics, such as density functional theory (DFT) based methods. Professor Bueno acted as academic and researcher visitor in several European universities such as Universities of Valence and Jaume I (Spain), University of Paris (France) and University of Oxford (UK). Since 2015 he is invited member of American Chemical Society (ACS), Research Fellow Director of the Royal Society (https://royalsociety.org/people/paulo-roberto-bueno$12682 /$ ) and Fellow of the Royal Society of Chemistry (FRSC). He has being engaged with technological transfer and has serve as due-diligent consultant for USA and European companies. He is one of the founders of Oxford Impedance Diagnostics (http://oxfordimpedance.com/).
Dr. Bedatty Fernandes is currently occupying a post doctorate position at Nanobionics research group (www.nanobionics.pro.br) at São Paulo State University, UNESP He published 12 paper in the fields related of analytical chemistry, environmental chemistry and electrochemical biosensors. During execution of his Ph.D. project, he was engaged in the development of a totally new label-free diagnostic assay using electrochemical capacitance methodologies. Also during the execution of his Ph.D. project, he additionally was a contributor for 2 patents (licensed to Oxford Impedance Diagnostics). For his thesis work in chemistry he received, in 2015, an honorable mention from CAPES (Coordination for the Improvement of Higher Education Personnel, a governmental agency in Brazil associated with the ministry of education). Nowadays, his main scientific interest is focused on the application and development of analytical methods involving electron charge transfer and storage occurring in nanoscale (molecular) and supra-molecular (bio-molecular) structures. 